\title{
Arterial Viscoelastic Model using Lumped Parameter Circuit With Fractional-Order Capacitor
}

\author{
Mohamed A. Bahloul and Taous Meriem Laleg-Kirati \\ King Abdullah University of Science and Technology (KAUST) \\ Thuwal 23955-6900, Makkah Province, Saudi Arabia \\ E-mail:\{mohamad.bahloul, taousmeriem.laleg\}@ kaust.edu.sa.
}

\begin{abstract}
Cardiovascular diseases are deemed to be the underlying source of mortality globally. Modeling the systemic arterial system is of a vital importance in the diagnosis and assessment of cardiac pathophysiology. In this work, we explore the fractional viscoelastic properties of the arterial blood vessel, and present a fractional-order lumped parameter model. We refer to this model as arterial fractional order visco-elastic (AFV) model. A novel feature of this characterization is that the ideal analog parameter displaying the arterial compliance in the wellknown windkessel model, has been replaced by a fractional order element (Fractional Capacitor). It displays the complex and frequency dependent total arterial compliance by combining both resistive and capacitive properties that exhibit the fractional viscoelastic behavior of the vessel wall. The contribution of both characteristics is controlled by a fractional differentiation order parameter $(\alpha)$ enabling an accurate and real physiological description. The proposed model offers a pioneer way for the better interpretation of the viscoelastic effect on the arterial hemodynamic. In addition, it can be investigated as a reliable computer-based simulation platform for cardiac pathophysiology diagnosis and treatment.

Keywords-Cardiovascular system, Windkessel model, Viscoelasticity, Hemodynamic, Fractional capacitor, Fractional Calculus.
\end{abstract}

\section{INTRODUCTION}

Over the last century, many research efforts have been invested to model the arterial hemodynamic variables such as blood flow (BF) and pressure (BP). Their mission was to make use of an accurate computational model to identify and estimate parameters difficult to measure invasively. Windkessel (WK) lumped parametric model is one of the most commonly used 0-Dimension (No space variation) model that describes the systemic arterial system. It characterizes the after-load faced by the heart when pumping the blood through the systemic arterial network. It combines electrical components in a simple and elegant way, such as resistor, inductor, and capacitor to link the $\mathrm{BF}$, and $\mathrm{BP}$ to the arterial resistance and compliance [1]. Several number of WK models have been proposed and extensively studied, over the last decades. They can be classified into elastic and viscoelastic Windkessel models. The elastic Windkessel considers that the arterial vessel is a pure elastic reservoir (represented by an ideal capacitor) which contrasts the natural behavior. In fact, similar to the most existing biological tissues, vascular vessel wall shows a viscoelastic property rather than pure elastic [2]. Several research attempts have tried to characterize this comportment by developing viscoelastic windkessel (VWK) models. VWKs are similar to the standard $\mathrm{WK}$, however, the ideal capacitor is replaced by a complex and frequency dependent capacitor. This latter complex compliance is developed based on the mechanical Voigt cell model whose electrical analogue is a resistor in series with a capacitor representing respectively the viscous and elastic comportments [2]. However, Roberto et al. [3] showed that modeling the arterial vessel motion using Voigt cell characterization is limited and does not certainly drive to a realistic representation of the vascular viscoelasticity. This is due to the fact that, Voigt cell model does not account for the stress-relaxation experiment. This issue can be addressed by increasing the viscoelastic model order which leads to a ladder network representation. However, adopting the ladder model conducts to another challenge represented in the huge number of parameters needed to be identified. Literally, the collected physiological data is usually not sufficient to identify all the variables of these complex models. Furthermore, it is well knowing that reduced order models are preferable for their simplicity and their ease to characterize and use.

Over the last decade, many research work have showed the potential of describing the arterial wall viscoelasticity using fractional derivative (FD) modeling, reducing the number of parameters and exposing a natural response. Thence, a key missing item in such viscoelastic arterial lumped parametric representation, is a fractional-order analog component that can provide a reliable, realistic and reduced depiction of the real fractional viscoelastic behavior [4]. Recently, we have proved the ability of fractional order capacitor to describe the real behavior of the arterial compliance by proposing for the first time, a three-element fractional-order viscoelastic arterial windkessel model [5].

In this paper, we investigate the characteristic of fractional capacitor (FC) to model the arterial hemodynamic and the effect of viscoelastic properties on BP. The rest of the paper is organized as follows. Section II recalls some definitions of FD and FC. Section III describes the proposed arterial viscoelastic fractional model. In section IV, simulations of arterial BP are shown with discussion. Section V concludes the paper and outlines the future work. 


\section{PRELIMINARY}

\section{A. Fractional Order Capacitor}

Fractional order capacitor is an electrical component which is composed of two parallel plates confining a lossy material. It is also defined as a Constant Phase Element (CPE) whose equivalent impedance has a constant phase angle (between $0^{\circ}$ and $-90^{\circ}$ ) over the whole frequency band, from zero to infinity [6]. Its impedance in s-domain can be expressed as

$$
Z_{C F}(s)=\frac{1}{C_{F} s^{\alpha}} .
$$

For $s=j w, Z_{C F}$ can be described using the following equation:

$$
Z_{C F}(j w)=\underbrace{\frac{1}{C_{F} w^{\alpha}} \cos (\phi)}_{Z_{D}}-\underbrace{j \frac{1}{C_{F} w^{\alpha}} \sin (\phi)}_{Z_{S}}
$$

where $\phi=\alpha \frac{\pi}{2}$ and $C_{F}$ is the fractional-order capacitance whose unit is Farad $/ \mathrm{sec}^{1-\alpha}$. The Fractional impedance is variable with an exponent $\alpha(0<\alpha<1)$, which is the fractional differentiation order. $\alpha=1$ refers to an ideal capacitor with a phase angle equals to $-90^{\circ}$ and $\alpha=0$ to an ideal resistor whose phase angle equals to $0^{\circ}$. As we can notice from Eq. 2, $Z_{C F}$ can be split into $Z_{D}$ (real part) and $Z_{S}$ (imaginary parts). They represent respectively the dissipative and storage components which might exhibit the viscous and elastic parts of such viscoelastic system. In term of circuitry, fractional order capacitor is still hypothetical, however its feature empower it to be investigated for identifying behaviors of viscoelastic vascular tissue. Over the last two decays, many research efforts have been invested to approximate fractional-order capacitors by combining ideal elements in different ways. The simplest and most optimized one is by using a recursive association of resistance and capacitance known as Foster integer-order ladder $R C$ network [7]. It is worth to emphasize that this configuration is similar to the viscoelastic equivalent analog circuit model witch is based on ladder network viscoelastic representation.

\section{B. Fractional Derivative}

There are several definitions of fractional derivatives [8]. In this work we focus on the Grunwald Letnikov (GL) definition that define the fractional derivative of a given function $g$ as

$$
D_{q}^{t} g(t)=\lim _{h \rightarrow 0} \frac{1}{h^{q}} \sum_{i=0}^{\infty} c_{i}^{(q)} g(t-i h)
$$

where $h$ is the time step, $q$ is the fractional order and $c_{i}^{(q)}$ for $i=0,1, \ldots$ are the binomial coefficients that can be computed using the following expression,

$$
c_{0}^{(q)}=1, c_{i}(q)=\left(1-\frac{1+q}{i}\right) c_{i-1}^{(q)}
$$

In the case where we consider $n=\frac{t-a}{h}$ where $a$ is a real constant that express a limit values, Eq. 3 can be rewritten as:

$$
{ }_{a} \mathrm{D}_{q}^{t} g(t)=\lim _{h \rightarrow 0} \frac{1}{h^{q}} \sum_{i=0}^{[n]} c_{i}^{(q)} g(t-i h),
$$

Where a and t refers to the bounds of the operation for ${ }_{a} \mathrm{D}_{q}^{t} g(t)$ and [.] corresponds to the integer part.

The discrete approximation formula of the $q^{\text {th }}$ derivative at the points $k h(k=1,2, \ldots)$ has the following form:

$$
\left(k-L_{m} / h\right) \mathrm{D}_{q}^{t_{k}} g(t)=h^{-q} \sum_{i=0}^{k} c_{i}^{(q)} g(t-i h),
$$

where $L_{m}$ corresponds to the "memory length".

Hence, the general numerical solution of any the fractional order differential equation:

$$
{ }_{a} \mathbf{D}_{q}^{t} y(t)=g\left(y\left(t_{k}\right), t\right)
$$

can be written as follow:

$$
y\left(t_{k}\right)=g\left(y\left(t_{k}\right), t_{k}\right) h^{q}-\sum_{i=1}^{k} c_{i}^{(q)} y\left(t_{k-i}\right) .
$$

\section{Fractional Arterial Model}

The proposed fractional order model is similar to the well known viscoelastic windkessel model. However, we replace the complex and frequency dependent capacitor $\left(C_{c}(j w)\right)$ accounting for the total arterial compliance by a fractional order capacitor $\left(C_{F}\right)$. The later fractional element deems to present the real viscoelastic behavior of the blood vessel. This is due to the fact that, fractional order capacitor can bee seen as an equivalent lumped parametric element of an infinite number of Voigt cell connected in series, as illustrated in fig. 1 (a) and (b). The model parameters are $Z_{0}, C_{F}$ and $R$ respectively refers to the aortic characteristic impedance, fractional complex and dependent frequency compliance and the peripheral resistance as shown in fig. 1 (d). The proposed model comprises only one dynamic fractional element. Hence, only one state is needed to characterize the dynamic of the system. Assuming, the blood flow $F_{a}$ pumped from the heart to the whole arterial network is the input, thereby the state equation of the system by applying Kirchhoff's Current and Voltage laws (KCL \& KVL) to the left mesh of the circuit shown in fig. 1 (d) is as follow:

$$
\begin{gathered}
D_{\alpha}^{t} P_{p}(t)=-\frac{1}{R C_{F}} P_{p}(t)+\frac{1}{C_{F}} F_{a}(t) \\
P_{a}(t)=P_{p}(t)+Z_{0} F_{a}(t)
\end{gathered}
$$

Where $P_{a}(t)$ refers to the arterial pressure output. The state $P_{p}(t)$ denotes the pressure across the fractional arterial compliance. Applying Laplace transform and assuming null initial conditions, the fractional aortic input impedance $Z_{F}$ may be expressed as:

$$
Z_{F}(s)=\left(R+Z_{0}\right) \frac{1+s^{\alpha} \tau_{N}}{1+s^{\alpha} \tau_{D}}
$$




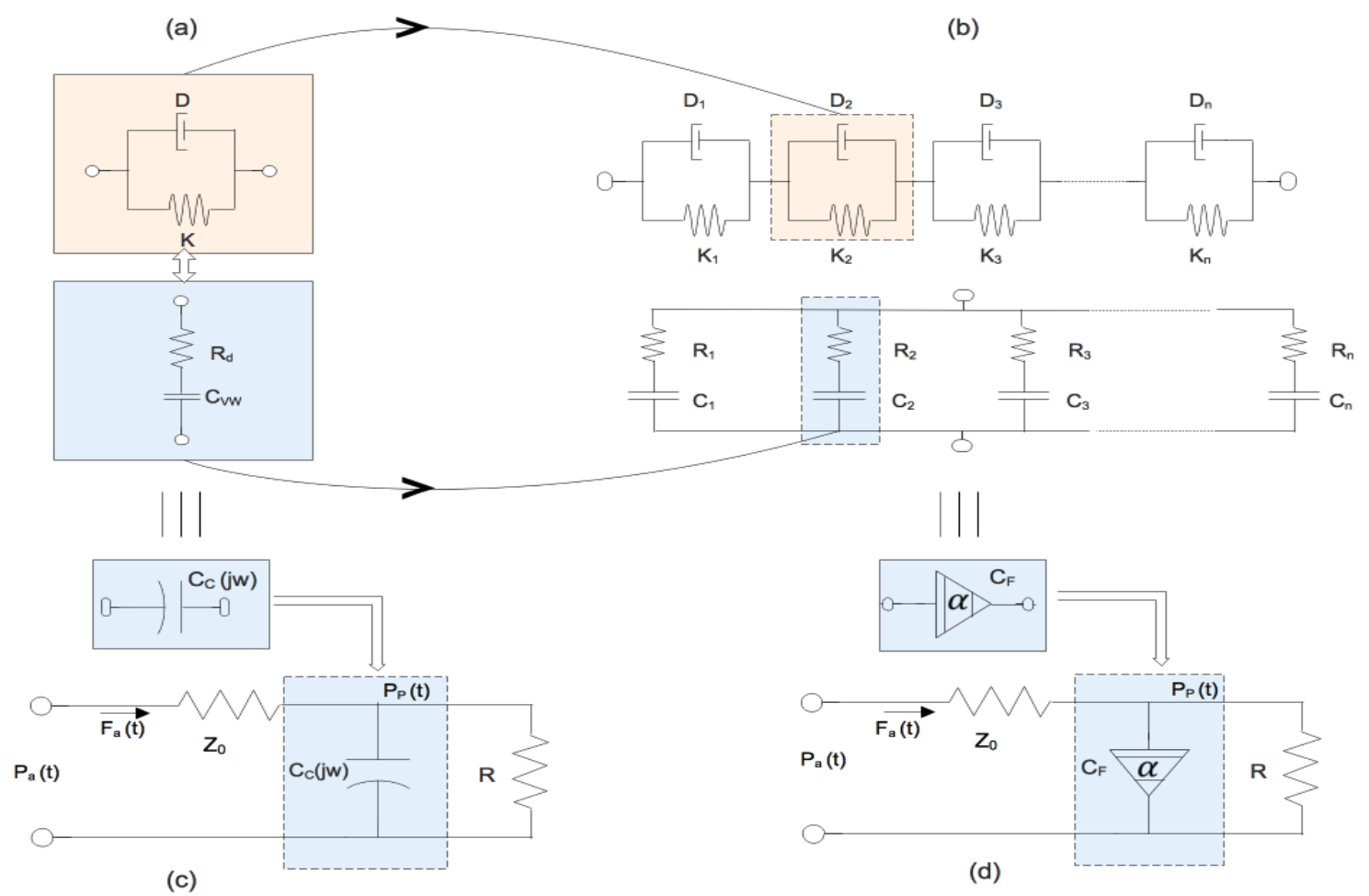

Fig. 1: (a) Viscoelastic Voigt Cell model consisting of a spring with elastic constant $K$ connected in parallel to a dashpot with a damping factor $D$ and its electrical analogue that consists of a resistor $R_{d}$ in series with a capacitor $C_{v w}$, (b) Viscoelastic ladder network comprising an infinite Voigt cells connected in series and its electrical analogue, (c) Viscoelastic Windkessel model analog circuit. This model consists of a resistance $Z_{0}$ representing the characteristic aortic impedance in series with the two element Windkessel model that comprises a complex capacitor $C_{C}(j w)$ connected to a resistor $R$ which exhibit the total arterial compliance based Voigt cell analogy and peripheral resistance respectively, and (d) Fractional Viscoelastic Windkessel model analog circuit. This model is similar to the Viscoelastic windkessel, whereas the arterial compliance is represented by a fractional capacitor $C_{F}$ equivalent to Voigt cell based ladder network.

where $\tau_{N}$ and $\tau_{D}$ are time constants and have the following expressions:

$$
\left\{\begin{array}{c}
\tau_{N}=\frac{R Z_{0}}{R+Z_{0}} C_{F}, \\
\tau_{D}=R C_{F} .
\end{array}\right.
$$

Thus the input impedance modulus and phase angle at a specific frequency $w$ is given by Eq. 13 and 14 .

\section{NUMERICAL RESULTS AND DISCUSSION}

GL approximation (Eq. 3) has been used to solve the fractional differential equations (Eq. 9) in MATLAB. The blood flow $F_{a}(t)$ across aorta has been used as an input signal. Physiologically, the cardiac cycle can be divided into two main phases systolic and diastolic. During the systolic phase, the ventricle in the heart contracts and pumps blood out to the arterial network. In the diastolic phase, the heart ventricles are relaxed and the heart fills with blood. Assuming that a cardiac cycle length is $T_{C}$. For the sake of simplicity, $F_{a}$ has been approximated to have Sine shape during the systolic phase, ( $\left.T_{S} \approx \frac{1}{3} T_{C}\right)$ and to be zero during the diastolic phase. The following equation represents $F_{a}$

$$
F_{a}(t)= \begin{cases}F_{0} \sin ^{2}\left(\frac{\Pi t}{T_{s}}\right) & t \in\left\langle 0, T_{S}\right\rangle \\ 0 & t \in\left\langle T_{S}, T_{C}\right\rangle\end{cases}
$$

In our simulations we choose $F_{0}=500 \mathrm{ml}$ and $T_{C}=$ $1 s$. We propose to examine the effect of fractional derivatives on BP, $P_{a}$ signal and the modulus-phase angle of the aortic input impedance $Z_{F}$. All the parameters values of the systemic arterial system were taken from [9] for a normotensive person.

Fig. 2 shows the numerical simulation of the arterial BP for different values of $\alpha$. It can be observed that as $\alpha$ decreases from 1 to 0.7 the blood pressure increases. For instance, the systolic pressure is around $160 \mathrm{mmHg}$ for $(\alpha=0.7)$, whereas

$$
\begin{gathered}
\left|Z_{F}\right|=\left(R+Z_{0}\right) \frac{\sqrt{\left(1+w^{\alpha} \tau_{N} \cos \left(\alpha \frac{\pi}{2}\right)\right)^{2}+\left(w^{\alpha} \tau_{N} \sin \left(\alpha \frac{\pi}{2}\right)\right)^{2}}}{\sqrt{\left(1+w^{\alpha} \tau_{D} \cos \left(\alpha \frac{\pi}{2}\right)\right)^{2}+\left(w^{\alpha} \tau_{D} \sin \left(\alpha \frac{\pi}{2}\right)\right)^{2}}} \\
\angle Z_{F}=\tan ^{-1}\left(\frac{w^{\alpha} \tau_{N} \sin \left(\alpha \frac{\pi}{2}\right)}{\left.1+w^{\alpha} \tau_{N} \cos \left(\alpha \frac{\pi}{2}\right)\right)}\right)-\tan ^{-1}\left(\frac{w^{\alpha} \tau_{D} \sin \left(\alpha \frac{\pi}{2}\right)}{\left.1+w^{\alpha} \tau_{D} \cos \left(\alpha \frac{\pi}{2}\right)\right)}\right)
\end{gathered}
$$



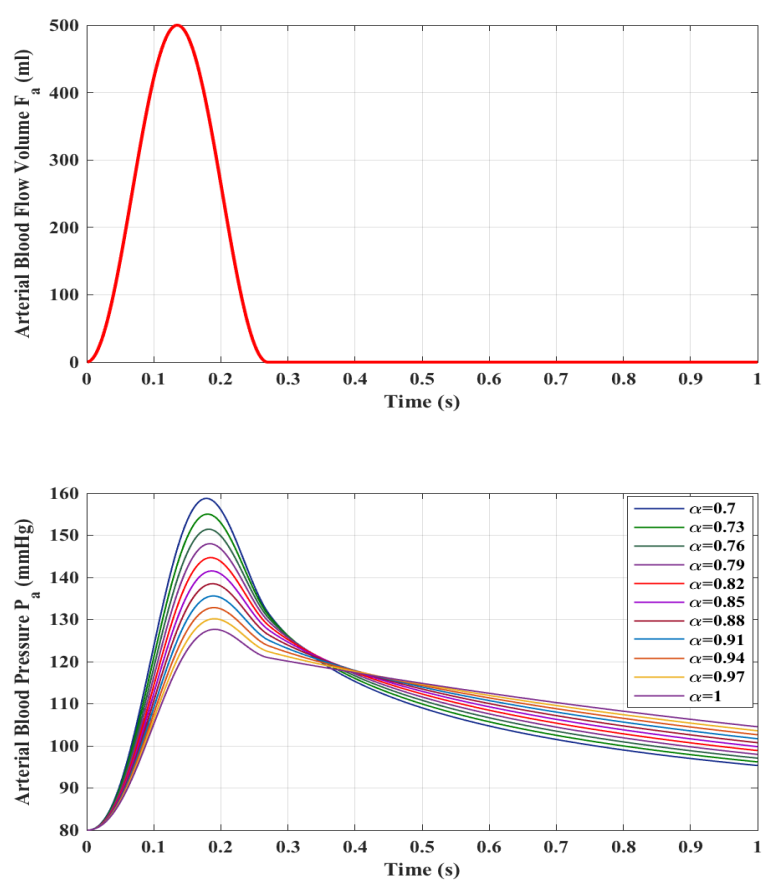

Fig. 2: Numerical simulation of the arterial blood pressure $P_{a}$ for different fractional differentiation order $\alpha$.

it is about $125 \mathrm{mmHg}$ for $\alpha=1$ which corresponds to the normotensive condition. Analytically, it can be derived from Eq. 2 that a decrease in the fractional order parameter $\alpha$ leads to a increase in the vessel wall resistivity. Furthermore, It is known in cardiac-pathologies, that an increase in the vessel wall resistivity that causes a narrowing in the vessel body channel (known as stenosis) conducts the arterial system from normotensive state to hyper-tensive state.

Aortic input impedance (AII) represents a simple and comprehensive resource for hemodynamic parameters evaluation and vascular pathologies identifications. In addition, from AII plots, it is easy to extract the different arterial parameters such as compliance, characteristic impedance and peripheral resistance. Hence, get a sufficient idea about the arterial system state. Fig. 3 shows the effect of varying $\alpha$ from 0 to 1 on the modulus-phase angle of the AII.

Based on this results, it is worth to deduce that the fractional order element not only leads to an accurate estimation of the viscoelastic properties of the vessel motion but, also help to get an improved analysis of the vessel wall resistivity variation effect. Thus, the arterial viscoelastic level described by $\alpha$ could help to understand the mechanism through which the arterial system hemodynamic behavior changes with the changes of vessel tissue properties.

\section{CONCLUSION}

Modeling the arterial hemodynamic is of a vital importance. The existing lumped parametric model are limited in term of viscoelastic effect interpretation. In this paper, the use of a fractional order capacitor to model the real behavior of the total arterial compliance has been investigated. The simulated blood pressure and modulus-phase angle of the aortic input impedance have been studied for different
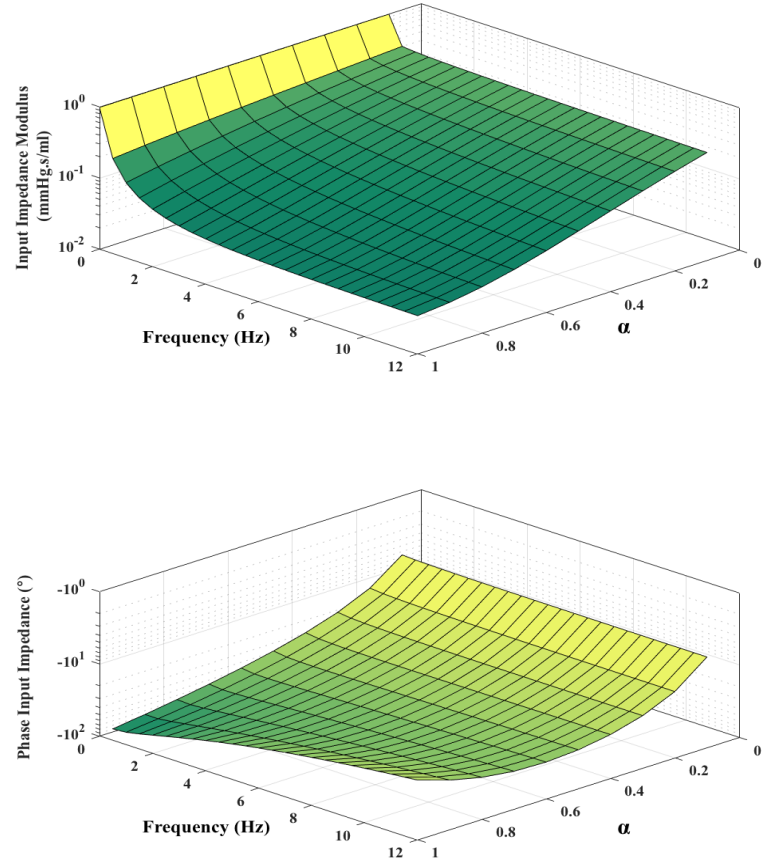

Fig. 3: Numerical simulation of the modulus and phase angle of the aortic input impedance versus the fractional differentiation order $\alpha$ for the proposed model.

fractional differentiation order $\alpha$ that refers to the variation of the level of blood vessel wall viscosity.

In future work, real data sets will be used to investigate and validate the model. Also, the identification of the fractional differentiation orders using real blood pressure and modulusphase angle data for normal and pathological conditions will be studied.

\section{REFERENCES}

[1] N. Westerhof, J.-W. Lankhaar, and B. E. Westerhof, "The arterial windkessel," Medical \& biological engineering \& computing, vol. 47, no. 2, pp. 131-141, 2009.

[2] R. Gul, "Mathematical modeling and sensitivity analysis of lumpedparameter model of the human cardiovascular system," Ph.D. dissertation, Freie Universität Berlin, 2016.

[3] R. Burattini and S. Natalucci, "Complex and frequency-dependent compliance of viscoelastic windkessel resolves contradictions in elastic windkessels," Medical Engineering and Physics, vol. 20, no. 7, pp. 502-514, 1998.

[4] D. Craiem, F. J. Rojo, J. M. Atienza, R. L. Armentano, and G. V. Guinea, "Fractional-order viscoelasticity applied to describe uniaxial stress relaxation of human arteries," Physics in Medicine \& Biology, vol. 53, no. 17 , p. 4543, 2008.

[5] M. A. Bahloul and T. M. Laleg-Kirati, "Three-element fractional-order viscoelastic arterial windkessel model," in Engineering in Medicine and Biology Society (EMBC), 2018 40th Annual International Conference of the IEEE. IEEE, 2018.

[6] A. S. Elwakil, "Fractional-order circuits and systems: An emerging interdisciplinary research area," IEEE Circuits and Systems Magazine, vol. 10 , no. 4 , pp. 40-50, 2010.

[7] J. Machado, "Discrete-time fractional-order controllers," Fractional Calculus and Applied Analysis, vol. 4, pp. 47-66, 2001.

[8] L. Dorcak, "Numerical models for the simulation of the fractional-order control systems," arXiv preprint math/0204108, 2002.

[9] T. Kind, T. J. Faes, J.-W. Lankhaar, A. Vonk-Noordegraaf, and M. Verhaegen, "Estimation of three-and four-element windkessel parameters using subspace model identification," IEEE Transactions on Biomedical Engineering, vol. 57, no. 7, pp. 1531-1538, 2010. 